\title{
CAA Approaches for Duct Elements of HVAC Systems
}

\author{
Pavel Drabek ${ }^{1, *}$, and Martin Zalesak ${ }^{1}$ \\ ${ }^{1}$ Tomas Bata University in Zlin, Faculty of Applied Informatics, Department of Automation and \\ Control Engineering, Nad Stranemi 4511, 76005 Zlin, Czech Republic
}

\begin{abstract}
In recent years there has been a significant increase in computing performance. This has allowed companies to make more use of numerical simulation methods during the development phase, even for areas that until recently were almost unfeasible. This article presents the fundamental aspects related to Computational Aero-Acoustics for internal flow with a focus on HVAC elements. The aim was to answer questions about the computational mesh, computational models and boundary conditions.
\end{abstract}

\section{Introduction}

HVAC Acoustics in buildings is still a relatively hot topic. Most attention is now focused on the steps to make the fan units operate most of the time in the energy-efficient area [1]. This approach also has a positive effect on the amount of generated noise of mechanical origin, which can be additionally reduced by a duct silencer [2]. Subsequently, the noise transmitted to the room is generated primarily due to the turbulent flow and solids in crossflow. For this reason, it is assumed that the optimization of the shaped parts of the duct elements, in order to reduce the pressure loss and the regenerated noise will be soon more discussed topic.

Recently, virtual prototyping methods have been used for such tasks [3]. The reason is a very significant increase in computing performance in recent years. Therefore, in addition to experimental methods, numerical simulations such as Computational Aero-Acoustics (CAA) have also become very attractive. However, currently, there are no unique solutions to all acoustic problems $[4,5]$. Instead, various strategies have been developed, but one that applies to one type of task may fail for another [3].

This paper discusses possible CAA approaches that may be applicable to internal flow in an HVAC system. The duct elements can be formed by various parts such as smooth pipes, perforated sheets, tilting flap shutters, grilles, perforated metal baffles filled with acoustic material and more. Some of these parts may require setting the wall boundary conditions, which means in practice a suitable choice of the computational model. The article discusses selected Scale Resolving Simulations (SRS) models, mesh requirements and 2D/3D solution, their limitations and uses.

\footnotetext{
* Corresponding author: pdrabek@utb.cz
} 


\section{Some Aspects Relevant to CAA}

CAA deals with the prediction of an aerodynamic noise source and its propagation numerically with the help of time-dependent equations [4]. Several approaches are commonly used to obtain the required acoustic information. Direct numerical simulation (DNS) is considered to be the most exact technique [6]. The main philosophy is to solve fully coupled compressible Euler and Navier-Stokes equations for the unsteady combined flow and acoustic field from the effective area down to the far-field observer. However, this technique is associated with high requirements for mesh size and computing time, as it is necessary to describe even the smallest vortices in the domain of interest [3]. These high requirements relate mainly to the area of low Mach numbers, where flow and acoustics represent a multi-stage problem with their own difficulties. These are due to the fact that the magnitude of the acoustic disturbances is several orders of magnitude smaller compared to aerodynamic forces. This large disparity between acoustic and flow variables presents a severe challenge to direct numerical simulation [7].

For the DNS, the biggest problem remains long-distance transmission, where the observer is relatively far from the sound generation location [5]. Therefore, for certain situations, it may be appropriate to combine DNS with methods using acoustic analogies. A possible solution offers so-called hybrid approaches, where the processes of sound generation and subsequent propagation are considered separately [8]. A nonlinear aerodynamic near field in which aerodynamic excitations generate sound is "approximated" to a linear acoustic far field in which there is no flow, and only sound propagates. The hybrid solution uses Scale Resolving models (SRS), as the turbulence generated by noise source cannot be extracted with accuracy from RANS simulations [5].

A typical scenario for solving a CAA problem is a two-phase computational approach [9]. In the first, only the aerodynamic part with fixed aerodynamic conditions is solved in order to obtain a state corresponding to a steady flow. The second phase is defined by an acoustic code operating with short periodic boundary conditions to affect the generation and propagation of acoustic waves.

\section{CAA for Duct Flows}

A special case of aerodynamic noise is the flow in the space bounded by the walls, such as ductwork. The generation of noise by a turbulent flow has a broadband sound expression that propagates along with the ductwork and eventually exits through the end distribution element into the inner protected space. Subsequently, the tonal contribution is usually due to cross flows at solid parts [10]. A suitable CAA method requires a more detailed discussion of the individual parts of the HVAC system.

In principle, it is not possible to perform a detailed CAA for the entire ductwork. Only individual parts are mainly considered. Such the most complex element for simulation is the silencer, whose attenuation capabilities are principally based on dissipative processes [11]. The reason is that such an element provides acoustic attenuation due to sound absorption material or sound radiation through the connection pipes. However, the silencer can also be considered as a sound source if it contains some pods [1]. Generally, the elements where several mechanisms of acoustic energy conversion are applied, it is challenging to achieve at least approximate results. This can only be achieved by appropriately entering the wall boundary conditions in the form of acoustic impedance or wall roughness. Best practices for circular duct silencer with a centre pod (see Figure 1) states in the following text. 


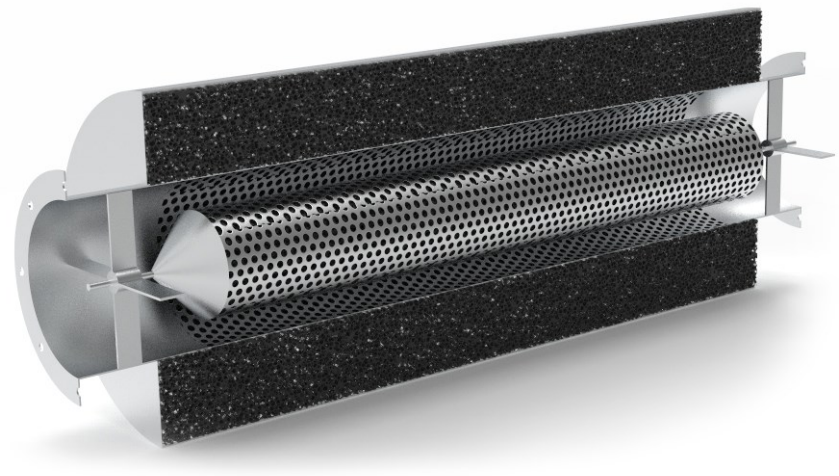

Fig. 1. Render of circular duct silencer with a centre pod GDE200.

\subsection{Mesh requirements}

The computational mesh has a major impact on the speed of calculation, convergence and quality of results. The appropriate mesh refinement is indicated by the maximum observed frequency and also by the selected type of spatial element. The relatively fine mesh must be maintained not only in places with unfavourable pressure gradients, for example near to profiles, but must also be maintained for free flows. In addition, this, combined with a very small time step, makes CAA a very time-consuming and hardware-intensive process.

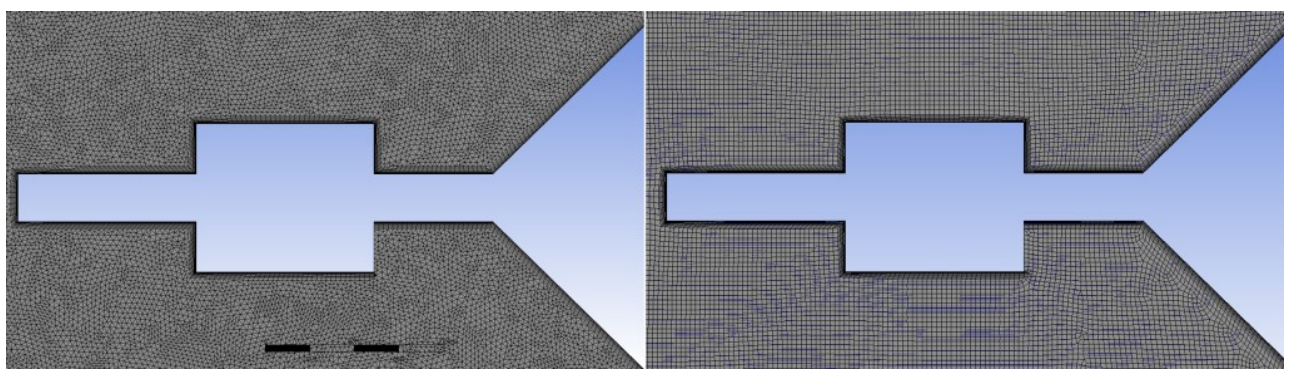

Fig. 2. Detail of a $2 \mathrm{D}$ computational mesh for a maximum observed frequency of $20 \mathrm{kHz}$ (left tetrahedral, right - hexahedral spatial element).

A relevant parameter is the number of mesh points required per wavelength to hold dissipation and dispersion within acceptable bounds. Recommendations for most methods, to keep the phase error within $1 \%$ accuracy, must be $20-25$ mesh points for the shortest wavelength [12]. The influence of the spatial element type was verified for the $2 \mathrm{D}$ solution in this work as well (see Figure 4). A detailed view on the mesh whose sizing should allow to cover acoustic signals for $f_{\max }=20 \mathrm{kHz}$ is shown in Figure 2, and its parameters are listed in Table 1. As can be seen, for defined frequency range, the mesh is exceedingly fine over the entire CAA domain.

To evaluate the mesh suitability for turbulence modeling, it is appropriate to perform a sensitivity analysis [13]. For CAA over a wide range of frequencies, the emphasis is on the velocity profile in the boundary layer at the wall. It is crucial to choose the proper height of the first layer so that the first node does not fall outside the boundary layer area. Otherwise, the wall functions used by our turbulence model may incorrectly calculate the flow properties at this first calculation point which will introduce errors into pressure drop and velocity results [13]. It is, therefore, appropriate to tune the individual prismatic layers to 
the $Y^{+}$value. It is also possible to adapt the mesh appropriately during the calculation by $Y^{+}$ value.

Table 1. Computational mesh parameters for 2D.

\begin{tabular}{|l|c|c|c|c|c|}
\hline Mesh Type & $\begin{array}{c}\text { Max. size } \\
{[\mathbf{m m}]}\end{array}$ & $\begin{array}{c}\text { First Layer } \\
\text { Height }[\mathbf{m m}]\end{array}$ & $\boldsymbol{f}_{\text {max }}[\mathrm{Hz}]$ & Time step [s] & $\begin{array}{c}\text { Number of } \\
\text { Cells [-] }\end{array}$ \\
\hline Tetrahedral & 0.8 & 0.064 & 20000 & 0.000025 & 1121878 \\
\hline Hexahedral & 0.8 & 0.064 & 20000 & 0.000025 & 585973 \\
\hline
\end{tabular}

In addition to other mesh quality metrics, it is necessary to verify the suitability of the mesh sizing for the selected time step. This is evaluated using the Courant-Friedrichs-Lewy criterion (CFL), which indicates a certain ratio of spatial to a temporal solution. The stability of the solver also depends on this ratio. If this criterion is exceeded, an incorrect solution occurs and probably a numerical divergence. For Large Eddy Simulation method (LES), a CFL near 1 is recommended [3].

\subsection{Methodology and Solver Settings}

In general, it is possible to solve CAA tasks themselves in 2D and 3D space. As mentioned above, a typical scenario for CAA is a two-phase calculation. This solution concept aims to speed up the overall calculation. The first phase represents a "fast" stationary calculation, which obtains the average velocity and pressure fields and other parameters, which further serve as an input boundary condition for the subsequent non-stationary simulation. This phase is also referred to as the RANS solution. For this type of internal flows, the realizable k- $\varepsilon$ model with enhanced wall function has proved to be the best option in terms of convergence.

The second phase begins with already properly developed fluid flows. Because almost all Scale Resolving models (SRS) assume unstable turbulent flow, it is necessary at first to generate turbulence from the averaged solution. This is done by synthetic turbulence generators, which ensure relatively realistic initial fluctuations. Subsequently, during the non-stationary calculation, the resulting turbulent structures are further developed. The individual phases of the calculation are depicted in Figure 3.

In the case of 3D CAA solutions, it is very easy to meet their limits. Large models or a wide range of frequencies can be a challenge even for today's high-performance computing servers. Therefore, for symmetrical models, it is appropriate to initially perform a 2D simulation instead of $3 \mathrm{D}$, for which no significant compromises need to be made. However, it should be noted that this solution does not allow accurate prediction of the absolute values of acoustic quantities. It can only be used to observe trends, for example, to compare multiple variants. Another difference between $2 \mathrm{D}$ and $3 \mathrm{D}$ solutions is the fact that for $2 \mathrm{D}$ CAA, it is necessary to operate with the so-called source correlation length. It is a parameter that is intended to compensate for the absence of the third dimension in a suitable way. This parameter needs to be estimated based on previous experience. This relatively reduces the reliability of the $2 \mathrm{D}$ solution.

Another issue previously mentioned is the areas covered with perforated sheets. Absorption silencers can again be an example. The presence of these surfaces in the air stream obviously contributes to the pressure drop of the element. Using the LES approach requires the pattern of holes modelling directly. However, due to the complexity of this approach, it is easier to replace these surfaces with a wall boundary condition determining the surface roughness, which allows only hybrid RANS-LES models. There are a large number of possible models; however, relatively recent studies $[14,15]$ have shown a clear 
predominance of the Stress-Blended Eddy Simulation (SBES) with Wall-Adapting Local Eddy-viscosity(WALE) model for most applications. The setting of the roughness parameters (roughness constant and height) is best determined from experimentally measured values of pressure loss. The set parameters must be constant for the entire flow range. Therefore, these parameters are most often determined iteratively.

If it is desired to include acoustic attenuation in the calculation due to the action of the absorbent material or radiation through the walls, it is necessary to use an acoustic wave model. This model can be started only if the considered medium is an ideal gas. Impedance boundary conditions of that model allow the modelling of soft walls. However, it is necessary to specify the reflection coefficient, which can be obtained from the experimental analysis.

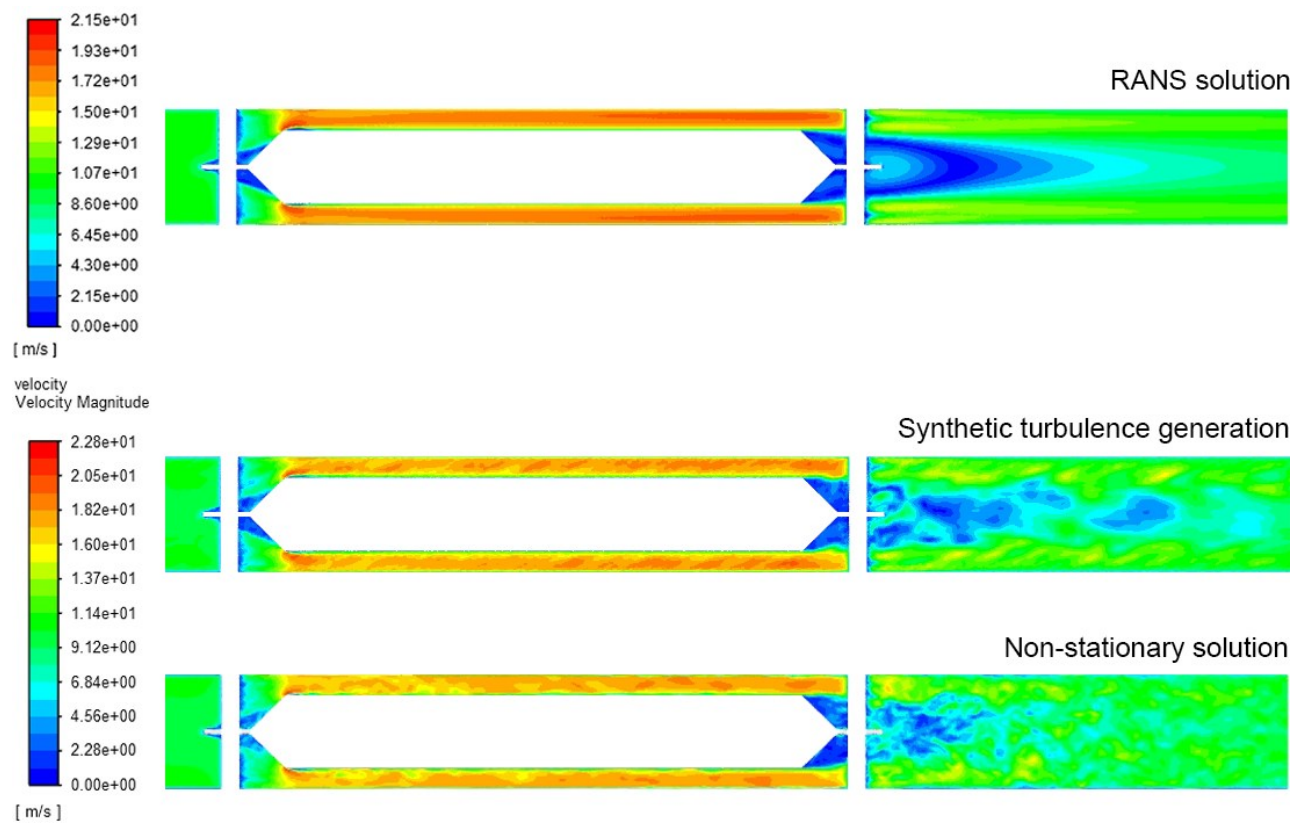

Fig. 3. Concept of CAA - Transition from a stationary to a non-stationary type of numerical simulation.

The recommended solver settings for the non-stationary part of the 3D CAA are given in Table 2. If the solution converges well, consider using non-iterative time advancement with fractional step for pressure-velocity coupling. This can significantly speed up the calculation.

Table 2. Solver settings for non-stationary part of 3D CAA.

\begin{tabular}{|c|c|c|c|c|}
\hline Type & $\begin{array}{c}\text { Pressure- } \\
\text { Velocity } \\
\text { Coupling }\end{array}$ & $\begin{array}{c}\text { Transient } \\
\text { Formulation }\end{array}$ & $\begin{array}{c}\text { Time } \\
\text { Discretization }\end{array}$ & $\begin{array}{c}\text { Pressure } \\
\text { Discretization }\end{array}$ \\
\hline Pressure based & SIMPLEC & $\begin{array}{c}\text { Bounded } \\
\text { second order } \\
\text { implicit }\end{array}$ & $\begin{array}{c}\text { Least Squares } \\
\text { Cell Based }\end{array}$ & Second order \\
\hline Acoustics model & \multicolumn{3}{|c|}{ Ffowcs-Williams \& Hawking } \\
\hline
\end{tabular}




\section{Conclusion}

The possibilities of prediction of the absolute values of acoustic quantities using CAA is a very complex issue, especially for HVAC elements that also provide a damping function. For such complex elements, hybrid RANS-LES models prove to be a more suitable variant than LES alone. This is mainly because some elements may require setting the wall boundary conditions. Examples can be perforated surfaces used in conjunction with sound absorption materials.

The use of these methods in 2D space does not offer an exact solution for determining the numerical values of sound pressure, but rather to observe the trends. The reason is that for a planar space, it is necessary to operate with the so-called source correlation length, which compensates for the absence of the third dimension. This parameter is not only a spatially measurable value but must be competently estimated. Therefore, in terms of accuracy, it is better to use 3D CAA approach, despite the high demands on computing performance.

The aim of this work was to present the fundamental aspects related to CAA for internal flow. This is an area that is not yet as well known as CFD; however, with the constant development of hardware, it can be assumed that the CAA will be a highly relevant topic in the near future. An effort was also made in this work to compare the planar and axisymmetric type of 2D space for CAA purposes. The results for different types of mesh can be seen in Figure 4. It can be observed that the planar model shows a better agreement with the course obtained by the measurement. From this it can be concluded that the choice of axisymmetric space for non-stationary simulation of turbulent flow did not prove to be suitable. The problem is that the calculation takes place only on one half of the plane of symmetry, which does not make it possible to capture the formation of vortices, which becomes asymmetric from a certain value of Reynolds number $(R e)$. It has also been shown that the shape of the spatial element of the computational mesh does not have a significant effect on the CAA results. Some defect can be expected at the upper limit of the observed frequency range. It is much more essential to adhere to the required dimension of network cells.

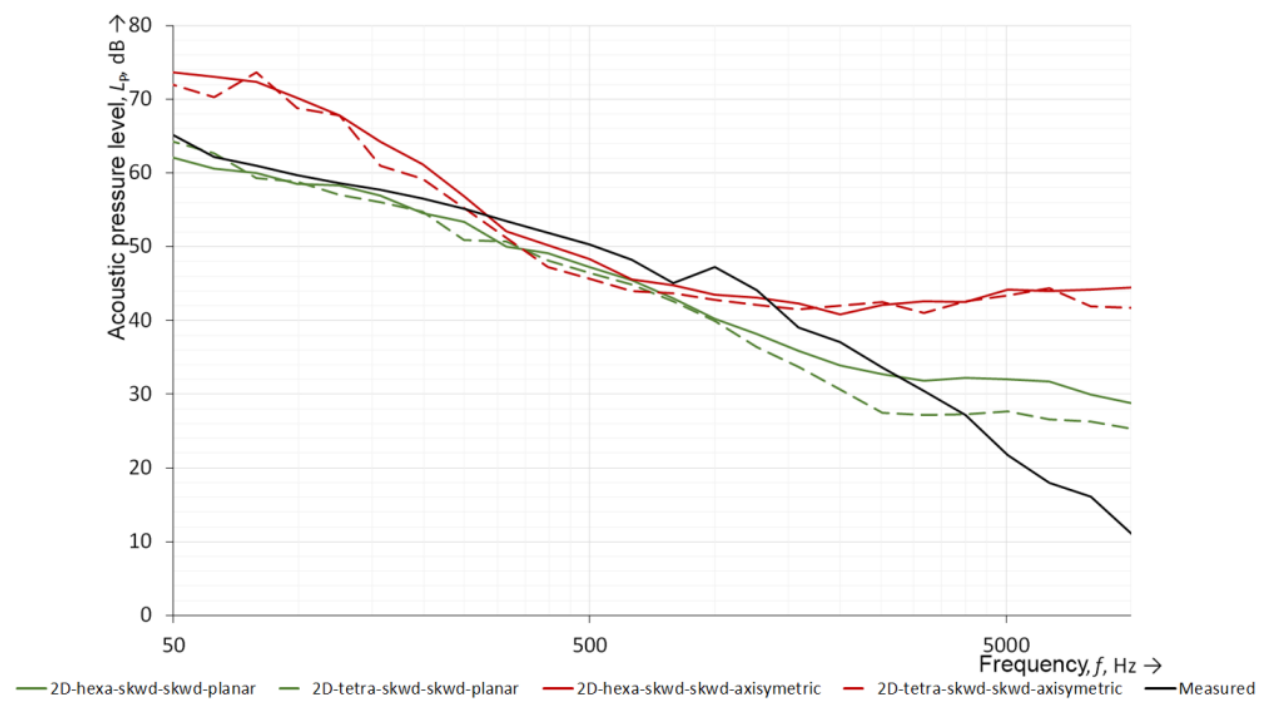

Fig. 4. Frequency curves of sound pressure levels for different types of 2D space and spatial element of mesh. 
This work was supported by the European Regional Development Fund under the project CEBIATech No. CZ.1.05/2.1.00/03.0089 and also by the internal research project of RVO UTB for year 2020.

\section{References}

1. Noise Control, Engineering Guide (2014)

2. A. Bhatia, B. Overview of Noise Control and HVAC Acoustics in Buildings (2012)

3. F. Menter, Best Practice: Scale Resolving Simulations in ANSYS CFD (2015)

4. T. Bose, Springer Aerospace Technology Book, 7 (2012)

5. C. Wagner, T. Hüttl, P. Sagaut, Cambridge University Press, 20 (2007)

6. A. Uzun, G. Blaisdell, A. Lyrintzis, 8th AIAA/CEAS Aeroacoustics (2002)

7. Ch. K. W. Tarn, AIAA Journal, 33, 10 (1995)

8. P. Quéméré, P. Sagaut, International Journal for Numerical Methods in Fluids, 40, pp. 903-925 (2002)

9. P. L. Roe, Technical prospects for computational aeroacoustics (1992)

10. C. M. Mak, Applied Acoustics, 63, pp. 81-93 (2002)

11. J. Delfs, Grundlagen der Aeroakustik (2016)

12. J. Thomas, P. Roe, In 11th Computational Fluid Dynamics Conference (1993)

13. H. Schlichting, K. Gersten, Boundary-Layer Theory (2017)

14. R. Florian, J. S. Menter, M. Gritskevich, In Progress in Hybrid RANS-LES Modelling, pp. 15-28 (2012)

15. J. Fröhlich, D. Terzi, Progress in Aerospace Sciences, 44, pp. 349-377 (2008) 